\title{
Genèses littéraires dans la correspondance de Pietro et Alessandro Verri
}

\section{Pierre Musitelli}

\section{(2) OpenEdition}

9 Journals

Édition électronique

URL : https://journals.openedition.org/genesis/4614

DOI : 10.4000/genesis.4614

ISSN : 2268-1590

Éditeur :

Presses universitaires de Paris Sorbonne (PUPS), Société internationale de génétique artistique littéraire et scientifique (SIGALES)

Édition imprimée

Date de publication : 15 décembre 2019

Pagination : 87-100

ISBN : 979-10-231-0650-3

ISSN : $1167-5101$

\section{Référence électronique}

Pierre Musitelli, «Genèses littéraires dans la correspondance de Pietro et Alessandro Verri », Genesis

[En ligne], 49 | 2019, mis en ligne le 01 décembre 2020, consulté le 12 janvier 2023. URL : http:// journals.openedition.org/genesis/4614; DOI : https://doi.org/10.4000/genesis.4614 


\title{
Genèses littéraires dans la correspondance de Pietro et Alessandro Verri
}

\author{
Pierre Musitelli
}

$\mathrm{C}$ ette étude examine la façon dont se mit en place, dans la correspondance de Pietro et Alessandro Verri, un système de transmission, d'annotation et de révision de leurs manuscrits respectifs qui institua ces échanges épistolaires trentenaires (1766-1797) en un laboratoire privé mais non solitaire de l'écriture. Leurs lettres ne se contentent pas d'informer sur le processus de création littéraire : elles font partie intégrante du processus génétique, auquel elles servent de cadre et de support. La correspondance bihebdomadaire des deux frères accueille des avant-textes - usage original ${ }^{1}$ et périlleux qui assujettit la composition des œuvres aux aléas et aux rythmes d'une communication par voie postale : les lettres arrivent souvent détrempées à l'automne et sont bloquées durant l'hiver par les neiges des Apennins. «Je n'ai pas de nouvelle de l'arrivée de ton manuscrit. Il y a tant de fleuves, de torrents et de ravins entre nous qu'il faut être patient ${ }^{2}$ », écrit Alessandro en novembre 1772, ne sachant si le manuscrit des Idee sull'indole del dolore e del piacere est arrivé à bon port.

L'examen portera aussi sur la langue de ces échanges et les mots qu'y emploient les épistoliers pour définir l'esprit et la finalité de leur collaboration. Car ce processus éditorial se joue dans le cadre d'une relation fraternelle et «sentimentale», pour utiliser l'adjectif forgé en 1768 par Laurence Sterne : une langue contaminée par des affects et des circonstances privées qui donnent à ce système de révision une tonalité et une intensité singulières. Entamée à l'automne 1766 au moment du départ d'Alessandro Verri et de Cesare Beccaria pour Paris, où l'opuscule Des délits et des peines fraîchement traduit en français connaissait un succès considérable, la correspondance des frères Verri s'établit à partir du printemps 1767 entre Milan, où réside Pietro, et Rome, où Alessandro s'est définitivement installé au terme de son périple européen.

Juriste et homme de lettres, administrateur de la Ferme puis conseiller au Département des finances de Lombardie,
Pietro Verri (1728-1797) achève en 1771 ses Meditazioni sulla economia politica qui closent une importante période de réflexion consacrée à l'économie et à la réforme des finances lombardes. Apprécié du jeune Condorcet et salué plus tard par Joseph Schumpeter comme l'un des grands théoriciens de l'économie politique du XviII ${ }^{\text {e }}$ siècle $^{3}$, Verri brigue, l'année où paraît ce texte, la présidence du Magistrato camerale, haute instance dirigeante des finances. Le poste lui échappe : amer, il prend ses distances vis-à-vis de l'administration publique, affirmant remiser les chimères de l'ambition pour donner un nouveau tournant à son expérience d'homme de lettres. Il ne sera plus le conseiller politique et l'économiste inquiet de prouver sa valeur aux yeux de l'administration austro-lombarde, mais le philosophe moraliste, soucieux du seul jugement de l'opinion. En 1772, il compose les Idee sull'indole del piacere e del dolore, parues en 1773 à Livourne. Ouvrage d'«économie morale» d'inspiration maupertuisienne, les Idee avancent que tous les plaisirs physiques et moraux ne consistent qu'en une rapide cessation de la douleur - le plaisir n'étant donc qu'une parenthèse dans l'existence des hommes. Kant, Leopardi et Stendhal seront des lecteurs attentifs de ce texte ${ }^{4}$.

Quant à Alessandro, qui était avec son frère l'un des contributeurs les plus prolifiques de la revue réformatrice Il Caffè parue à Milan de 1764 à 1766, il s'engage au cours des treize premières années de son séjour romain dans

1. Sur la rareté des correspondances qui éclairent les genèses à quatre mains, voir F. Leriche et A. Pagès, Genèse \& correspondances, Paris, Éditions des archives contemporaines/ITEM, 2012, p. 5.

2. Carteggio di Pietro e di Alessandro Verri dal 1766 al 1797, Milan, Cogliati/Milesi/Giuffrè, 1911-1942 [dorénavant Cart.], V, p. 221.

3. Voir P. Verri, Méditations sur l'économie politique, éd. A. Tiran, trad. A. Machet, M. Vitali-Volant et F. Manche, Paris, Classiques Garnier, 2015, «Vue d'ensemble», p. 10.

4. Voir C. Capra, I Progressi della ragione. Vita di Pietro Verri, Bologne, Il Mulino, 2002, p. 394. 
un travail d'expérimentation littéraire privé, éclectique et souvent novateur, que dévoilent sa correspondance et ses archives personnelles : traducteur de tragédies shakespeariennes, puis de Xénophon et d'Homère (Iliade), il abandonne son essai d'historiographie éclairée (Saggio sulla storia d'Italia) en cours de publication pour composer diverses tragédies, dont deux sont imprimées à Livourne en 1779 . C'est en 1781, au terme d'un nouveau revirement générique, qu'il fait paraître à Rome son premier roman, fantaisie antiquisante d'inspiration néoclassique, Le Avventure di Saffo poetessa di Mitilene, qui connaît en son temps un vaste écho.

\section{Une transposition épistolaire de la pratique collaborative des Pugni}

Pour comprendre l'origine de cette volonté de maintenir une pratique «plurielle» de l'écriture, malgré les délais et les risques inhérents aux transports postaux, il faut remonter aux années 1761-1766 au cours desquelles un cercle de jeunes aristocrates réformateurs, surnommé l'Accademia de'Pugni, l'Académie des coups de poing, publie coup sur coup trois manifestes des Lumières lombardes : les Meditazioni sulla felicità de Pietro Verri (1763), Dei delitti e delle pene de Cesare Beccaria (1764) et la revue Il Caffè, trois titres presque immédiatement traduits en français, quoique partiellement pour le dernier.

Une pratique collégiale de pensée et d'écriture prévaut dans le groupe des Pugni, dont témoignent les nombreux manuscrits préparatoires de l'Archivio Verri ${ }^{5}$ ainsi que la genèse complexe des Délits et des peines, patiemment reconstituée par Gianni Francioni6. Cette dernière offre un cas d'étude éclairant pour appréhender notre objet. Beccaria rédige un premier état manuscrit de son pamphlet dont Pietro prend l'initiative de modifier profondément la structure à l'occasion d'une seconde rédaction manuscrite dont il se charge seul. Francioni voit dans cette opération de recomposition une «violence » imposée à Beccaria ${ }^{7}$ et une victoire de «l'autorité et du prestige » de l'aîné des Verri sur la timidité du jeune auteur, placé dans une posture de disciple. Empruntant à la terminologie de George Thomas Tanselle, qui distinguait entre « vertical revision» (de nature à modifier le caractère et le contenu d'une œuvre) et «horizontal revision » (dont la seule finalité est d'améliorer ou d'achever le livre tel qu'il est conçu $)^{8}$, Francioni envisage d'abord de qualifier l'intervention de Verri de «révision verticale». Mais le fait que Beccaria ne rejette pas, dans ses propres révisions du second manuscrit puis dans les différentes éditions italiennes qu'il procure, les modifications introduites par Verri conduit Francioni à les considérer comme des «variantes approuvées par l'auteur» qui témoignent donc de la «volonté de l'auteur'»». Ainsi, en dépit de la «coercition psychologique» exercée par Verri sur Beccaria, et malgré l'importance des remaniements effectués qui confèrent au travail du premier tous les attributs d'une «révision verticale», les Délits offrent un exemple particulier de «livre en collaboration» dans lequel «la volonté de l'auteur et celle du réviseur ont fusionné», donnant naissance à « une nouvelle volonté d'auteur», à la fois hybride et unique ${ }^{10}$.

Le système dual de révisions qui s'instaure entre les frères Verri par lettres interposées est de nature plus sereinement «horizontale» car dépourvue de la «violence» qui marque les rapports entre Pietro Verri et Beccaria. L'addition de la volonté de l'auteur et de celle du réviseur donne aux textes leur physionomie finale, comme dans le cas des Délits. Mais, contrairement à l'ouvrage de Beccaria dont Pietro a fait entendre qu'il n'aurait pas existé sans lui11, jamais la paternité des œuvres nées de la collaboration des deux frères ne donne lieu à contentieux. Car chacun, sous couvert d'une rhétorique de l'amitié fusionnelle et de l'analogie d'esprit, s'acquitte, dans le processus de création et de révision, d'un rôle défini. Rêve d'identité et altérité fonctionnelle sont les principes moteurs de cette collaboration épistolaire.

5. Hébergé à Milan par la Fondazione Raffaele Mattioli per la storia del pensiero economico.

6. Voir C. Beccaria, Dei delitti e delle pene, éd. G. Francioni, Edizione nazionale delle opere di Cesare Beccaria, I, Milan, Mediobanca, 1984, «Nota al testo», p. 217-327.

7. G. Francioni, «Sulla violenza testuale. La "volontà" dell' "autore" fra libera espressione e assenso "coatto" », dans A. Postigliola (dir.), Pubblicare il settecento. Edizioni e ricerche in corso, Rome, Società italiana di studi sul secolo XVIII, 1991, p. 75.

8. G. Thomas Tanselle, «The Editorial Problem of Final Authorial Intention », Studies in Bibliography, n 29, 1976, p. 193.

9. G. Francioni, «Sulla violenza testuale», art. cit., p. 68.

10. Ibid., p. 76 (nous traduisons). Sur ces questions, voir également notre entretien avec Gianni Francioni dans ce même numéro.

11. Voir C. Capra, I Progressi della ragione, op. cit., p. 268. 


\section{Un témoignage sur l'acte créateur}

La première fonction de la correspondance relative à la création littéraire est maïeutique ou «électrisante», pour reprendre le néologisme qu'utilise Pietro dans une lettre à son frère de mars 1779: «Tu es le seul qui m'électrise et me pousse vers l'étude 12 ». Par le dialogue épistolaire, les deux frères s'animent l'un l'autre à surmonter leurs réticences, leur lassitude. Un an plus tôt, Alessandro adresse à son frère ces mots pleins d'énergie :

J'éprouve une indicible satisfaction lorsque je te vois revenir vers les lettres, et cette fois je te vois animé comme je le souhaite. Tu me trouveras toujours une torche à la main, non point pour t'éclairer, mais pour t'échauffer, presque jusqu'à t'embraser. Écris tout comme un torrent, toutes les idées qui se pressent en foule pour sortir des cavités de ton cerveau, n'en exclus aucune : grandes feuilles, encrier plein, couche tout par écrit, et puis l'on révise, et l'on choisit, mais le premier jet doit être hardi 13 .

La correspondance documente avec précision la phase de conception de certains textes, c'est-à-dire la phase préscripturale qui aboutit au «premier jet». En octobre 1772, Pietro dicte 14 en sept jours Idee sull'indole del piacere e del dolore à son secrétaire Giorgio Ghelfi. Il avait pris cette habitude (courante sous l'Ancien Régime) lors de la composition des Meditazioni sull'economia politica, dont la première version avait été dictée en moins de deux semaines à raison de « 4 heures par jour, environ, le soir ${ }^{15}$ ». Alessandro 1'y encourage en citant le Psaume 44 : «calamus scriba velociter scribentis ${ }^{16} »$, ( «[ma langue] est semblable à la plume d'un écrivain agile»). L'image de l'auteur sacré qui écrit sous la dictée de Dieu se transmue en celle de l'homme de génie dont l'inspiration s'affranchit de la «peine physique ${ }^{17}$ » de l'écrit. Pour Pietro, la voix est en effet le véhicule le plus immédiat d'une ferveur d'expression qui seule peut toucher la sensibilité du lecteur, tandis que la sédentarité du scripteur et la lenteur de sa main entravent le flux de sa pensée et conduisent à privilégier l'organisation logique des idées («connecter exactement les syllogismes 18 ») au détriment de leur vigueur. Il ajoutait, en octobre 1772 :

[...] lorsque Ghelfi est là, je me promène, je me couche, je suis en mouvement autant que je veux, et ma pensée va droit au but. [...] c'est ainsi que se forme un livre, que l'on lime ensuite, que l'on retouche à moindre peine ${ }^{19}$.

\section{Dès 1769 il affirmait à son frère :}

Tous les livres qui ont fait fortune en ce siècle sont écrits avec chaleur, et tous les progrès de la philosophie naissent d'écrivains enthousiastes ${ }^{20}$.

Pietro anticipe de quelques années Vittorio Alfieri qui donnera en Italie ses lettres de noblesse à la rhétorique du génie créateur, faisant du «sfogo», du «bollore» et de l' «impulso naturale » (c'est-à-dire à la fois l'épanchement et l'impetus de l'écriture) les piliers de sa poétique exposée dans Du Prince et des lettres (Del principe e delle lettere, 1778-1786). Alessandro, convaincu du bien-fondé de cette méthode, recourt à la même analogie :

Accouche; fais tout écrire; donne libre cours [lascia sfogo] à toutes tes idées ${ }^{21}$.

Je me réjouis que tu sois décidé à faire une œuvre de toutes les idées qui bouillonnent [bollono] dans ta tête ${ }^{22}$.

Quant à Pietro, il affirme que c'est la «fièvre» qui lui inspire le premier manuscrit, dicté, des Idee sull'indole 23 : écrire n'est donc pas un artisanat du style ; cela répond pour lui à une nécessité intérieure, à une passion, celle du bien commun. Son projet n'est pas d'être «auteur, simplement auteur ${ }^{24}$ », comme le confirmera la préface des Meditazioni sulla economia politica :

Je préfère infiniment le titre de bon citoyen, que j'estime plus glorieux, à celui de bon auteur, auquel je ne sais pas si j' aurais le droit de prétendre 25 .

12. Cart. X, 10 mars 1779 , p. 214. Je traduis moi-même toutes les citations, sauf mention contraire.

13. Cart. IX, 25 mars 1778, p. 247.

14. Sur la question de la dictée, voir J.-L. Lebrave, «Penser, dicter, écrire : pour une histoire des pratiques de composition», Romanic Review, vol. 86, 1995, $\mathrm{n}^{\circ} 3$, p. 437-450. Les considérations de Jean-Louis Lebrave évoquant la défiance de Quintilien pour l'écrit comme technologie d'invention, citant aussi la métaphore de la lime pour décrire l'emendatio convergent avec notre propos.

15. Cart. IV, 13 octobre 1770 , p. 21.

16. Ibid., 27 octobre 1770, p. 44.

17. Cart. V, 20 octobre 1772, p. 194.

18. Cart. X, 19 septembre 1778, p. 83.

19. Cart. V, 20 octobre 1772, p. 194.

20. Cart. II, 3 janvier 1769, p. 111.

21. Cart. IV, 24 octobre 1770 , p. 40.

22. Ibid., 27 octobre 1770, p. 44.

23. Cart. V, 25 novembre 1772, p. 214.

24. Cart. IV, 24 novembre 1770, p. 70.

25. Traduction de G. Mingard (1773), citée dans P. Verri, Méditations sur l'économie politique, op. cit., p. CCV. 


\section{Le processus génétique intégré aux échanges épistolaires}

La seconde fonction de la correspondance est génétique : l'échange épistolaire, en tant qu'espace de révision, constitue "une étape à part entière dans le processus rédactionnel $26 »$. Les dossiers génétiques des Meditazioni sulla economia politica (1770-1771) et des Idee sull'indole del piacere e del dolore (1772-1773)27 sont exemplaires, à cet égard, de la manière de procéder des frères Verri.

Une fois achevé le manuscrit d'enregistrement saisi par Ghelfi, Pietro commence une première phase de révision. C'est le travail de la «lime $28 »$, ainsi nommé en référence au limae labor horatien (Art poétique, v. 291) - image qui perdure dans toute la tradition littéraire italienne depuis le XIII ${ }^{e}$ siècle pour désigner l'activité de perfectionnement des textes, notamment poétiques. Pietro Verri reprend donc son manuscrit «depuis le début» afin de le «recomposer» en y intégrant tous les éléments qu'il avait écartés «pour ne pas perdre la chaleur des idées ${ }^{29}$ », mais sans presque jamais déplacer ni supprimer ce qu'il a déjà écrit 30 .

Le manuscrit est mis au propre et disposé à demimarge, dans la colonne de droite d'une page délimitée en deux par un pli central. Il est ensuite interfolié avant d'être relié : c'est-à-dire que des feuilles vierges de même dimension sont intercalées entre chaque feuille rédigée afin de recevoir les commentaires d'Alessandro. En tête de la première page blanche du manuscrit interfolié des Idee sull'indole transmises à Rome début novembre 1772, Pietro explique (fig. 1) :

Cher Sandrino, j' ai relié les feuilles blanches pour que tu y écrives tout ce qui te vient à l'esprit en lisant ce texte. [...] Ensuite, je ferai recopier les corrections par Ghelfi en marge du M.S. Je te saurais gré également d'écrire sur les feuilles en vis-à-vis, parce que j'aurai peu de temps à la fin de l'automne et que cela m'évitera d'avoir à comparer avec une feuille séparée. Écris comme tu le faisais précédemment : bien; obscur; faux; mauvais; élégant etc.

Alessandro lui répond en dessous :

J'écrirai tout ce qui me passera par la tête sans amour-propre.

Des consignes accompagnent toujours l'envoi du manuscrit depuis que les corrections par Alessandro du Piano per la Regia amministrazione delle finanze, à l'été 1770, n'avaient pas satisfait Pietro :

Je m'attendais de ta part à des critiques détaillées, qui fassent référence à des points du texte que j'ai numéroté à cet escient, afin que ton avis l'améliore avant qu'il ne sorte formellement de mes mains 31 .

Fin octobre 1770, alors qu'Alessandro révise pour la seconde fois le manuscrit de l'essai Sulle leggi vincolanti, Pietro annonce à son frère qu'il lui envoie le manuscrit d'une nouvelle œuvre, les Meditazioni sulla economia politica :

Tu examineras, tu feras tes critiques, tu me renverras le manuscrit avec tes critiques. Le manuscrit est disposé en colonnes ; tu pourrais faire des renvois en marge, avec des lettres ou des chiffres, et écrire tes pensées sur une feuille volante ou sur un petit quinterno ${ }^{32}$.

Pour conjurer le risque d'une perte du courrier, Pietro fait faire deux copies de son texte, ce qui lui permet de poursuivre ses propres corrections en attendant la réponse de son frère. À la réception du manuscrit, des assurances sont aussitôt données en réponse aux consignes et aux injonctions. Alessandro s'exécute en général avec humour : «Sabre au clair, fusil, baïonnette, pique, laisse-moi faire ${ }^{33}$ ». Pressé par son frère, il doit en général renvoyer ses premiers commentaires dès l'ordinaire suivant, sous trois à quatre jours. «À peine le manuscrit récupéré à la poste, je l'ai lu d'une traite», écrit-il à la réception des Idee; et il promet déjà :

Je te ferai donc quelques remarques et tu les auras sans faute au prochain ordinaire, écrites sur les feuilles blanches insérées, puisque je te renverrai ce même manuscrit par le courrier ${ }^{34}$.

26. Sur la façon dont un échange épistolaire peut relever «de la dynamique de l'avant-texte», voir F. Leriche et A. Pagès, Genèse \& correspondances, op. cit., «Avant-propos», p. 4.

27. La genèse de ces textes est présentée dans $I$ «Discorsi » e altri scritti degli anni Settanta, éd. G. Panizza, Edizione nazionale delle opere di Pietro Verri, III, Rome, Edizioni di Storia e Letteratura, 2004, «Nota ai Testi », p. 427-461 pour les Meditazioni, p. 462-472 pour les Idee.

28. Voir Cart. IV, p. 74 («lima e rilima più che puoi »); Cart. V, p. 194 et passim.

29. Cart. IV, 17 et 20 octobre 1770 , p. 25 et 28

30. Voir P. Verri, I «Discorsi », op. cit., « Nota ai testi », p. 434.

31. Cart. III, 22 septembre 1770 , p. 467.

32. Cart. IV, 31 octobre 1770 , p. 43 (quinterno: cahier de vingt pages).

33. Ibid., [14] novembre 1770, p. 66.

34. Cart. V, 11 novembre 1772 , p. 208-209. 


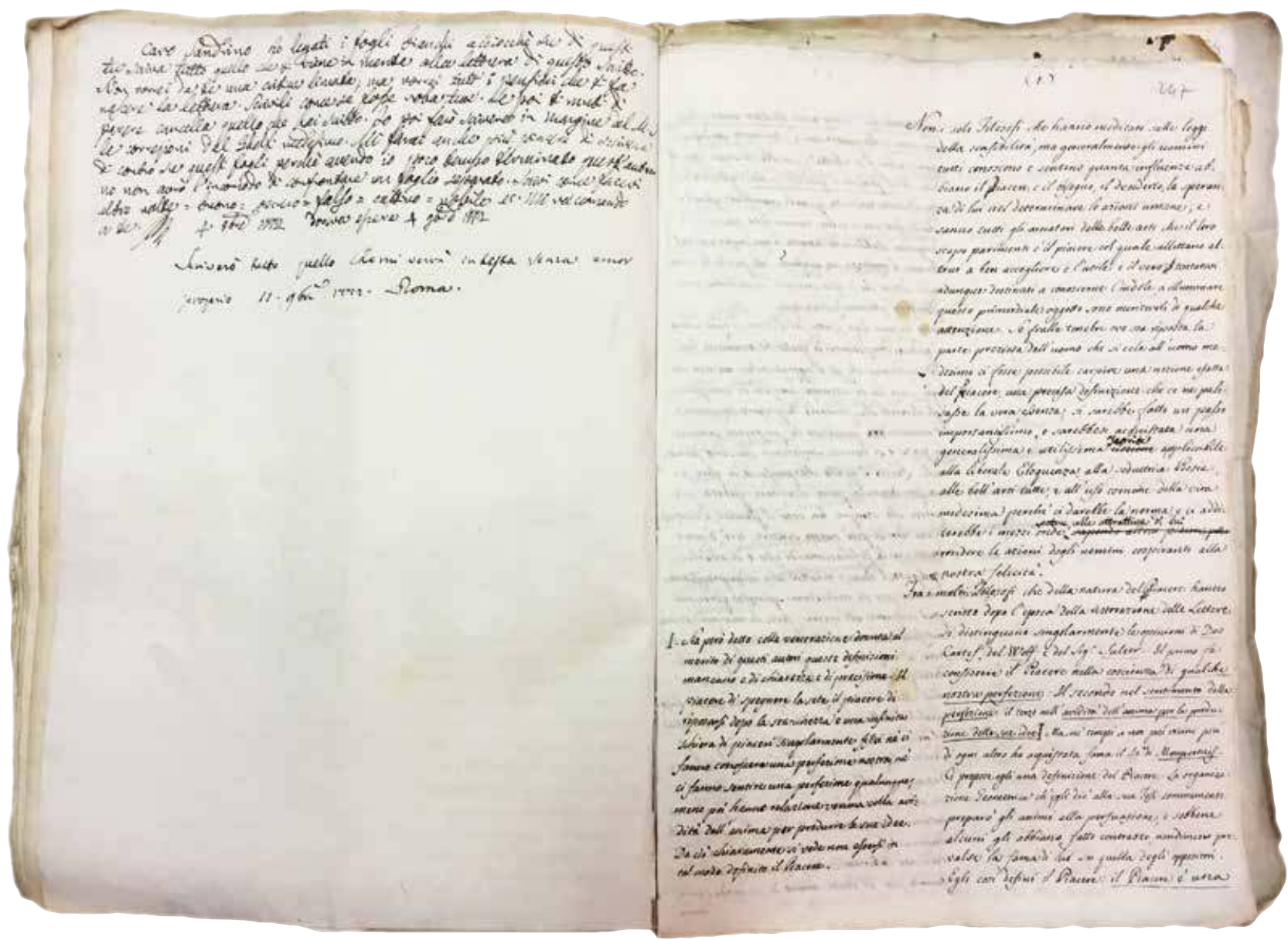

Fig. 1 : Archivio Verri, cartella 390.1, Idee sull'Indole del Piacere e del dolore, f. $246 \mathrm{v}^{\circ}-247 \mathrm{r}^{\circ}$.

Page de droite : le manuscrit de copiste avec ajouts et corrections autographes de Pietro Verri; page de gauche : première intercalaire avec en-tête de Pietro et réponse d'Alessandro. Milan, Fondazione Raffaele Mattioli per la storia del pensiero economico.

(C) Photographie de P. Musitelli, avec l'aimable autorisation de la Fondazione Raffaele Mattioli per la storia del pensiero economico

Trois jours plus tard, le manuscrit annoté est prêt à être renvoyé.

Différents supports sont utilisés simultanément ou successivement :

- Le manuscrit relié, tout d'abord : les espaces vierges du manuscrit interfolié sont amplement mis à profit par Alessandro (fig. 2), dont on peut parcourir aisément les indications autographes, sous lesquelles s'ajoutent dans de nombreux cas les commentaires et approbations de Pietro en réponse aux suggestions de son frère : «D'accordo»; «Accordato»; «Ho accomodato» (fig. 3). Un dialogue s'esquisse souvent dans ces marges, qui se poursuit dans les échanges épistolaires. On trouve des compliments, des marques d'empathie ou de tendresse : «Luminosamente espresso », juge Alessandro ; «Mi consoli», lui répond son frère (Archivio Verri, dossier 390.1, f. $260 \mathrm{r}^{\circ}$ ). «Bello», écrit encore le premier; «Caro Sandrino!», s'exclame le second (f. $266 \mathrm{v}^{\circ}$ ).
La colonne de gauche sert aux inserts et aux réécritures de certains paragraphes par l'auteur, qui apporte aussi des corrections à l'interligne selon les recommandations d'Alessandro. Il peut s'agir de corrections d'orthographe, de grammaire ou de syntaxe, de permutations de termes ou encore de corrections de «cacophonies» («queste questioni » devient «tali questioni », f. $294 \mathrm{v}^{\circ}-295 \mathrm{r}^{\circ}$ ). Pietro appose enfin près des remarques de son frère, qu'il entoure parfois pour les isoler, des indications à l'intention du copiste : «ne pas copier», «ne pas transcrire».

- Une fois restitué le manuscrit annoté, les révisions sont commentées dans les ordinaires qui suivent. L'auteur accepte, discute ou rejette librement les critiques de son frère, demande des précisions ou des approfondissements. Le réviseur répond aux observations, en adjoint de nouvelles. Puis l'auteur tranche. Souvent, comme l'ont montré les paratextes de l'édition nationale des œuvres 


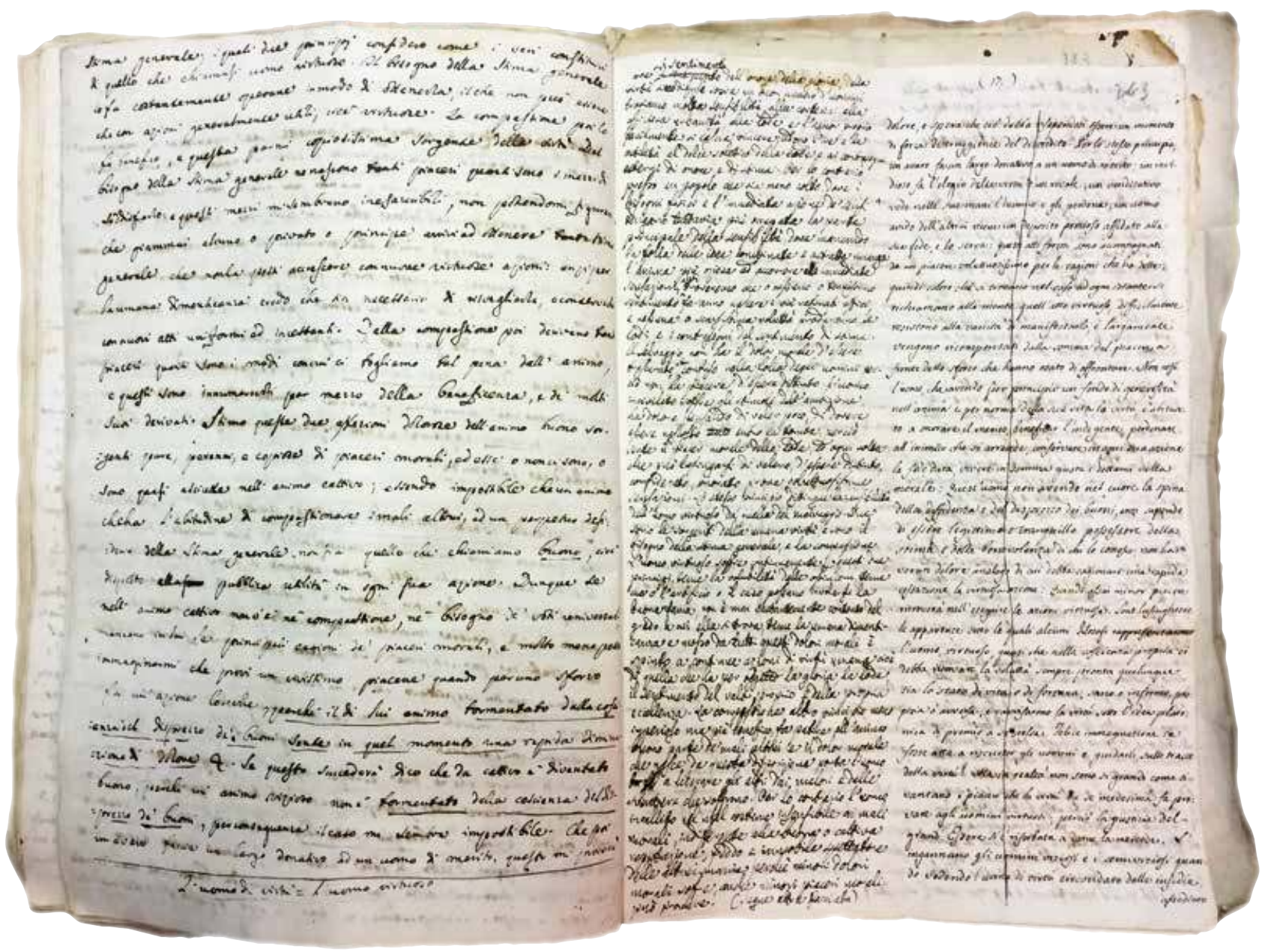

Fig. 2 : Archivio Verri, ibid., f. $262 \mathrm{v}^{\circ}-263 \mathrm{r}^{\circ}$

Page de gauche : suite d'un long commentaire commencé par Alessandro sur le recto précédent.

Page de droite : Pietro a barré d'un trait vertical le manuscrit de copiste dans la colonne de droite et recomposé son texte dans la colonne de gauche. Milan, Fondazione Raffaele Mattioli per la storia del pensiero economico.

(C) Photographie de P. Musitelli, avec l'aimable autorisation de la Fondazione Raffaele Mattioli per la storia del pensiero economico

de Pietro Verri (en particulier des Idee sull'indole et du Discorso sulla felicità, retravaillé en 1778), un grand nombre d'observations d'Alessandro sont intégrées au texte final. Ainsi, lorsqu' Alessandro relève dans le manuscrit des Idee un problème de logique argumentative (le raisonnement de Pietro semble aboutir à la conclusion que «l'homme mauvais est plus susceptible d'éprouver des plaisirs moraux, si bien qu'il vaut mieux être mauvais»), Pietro admet : «Je change tout et te remercie mille fois. Tu raisonnes avec évidence et je profite de tes raisonnements» (f. $264 \mathrm{r}^{\circ}$ : les amples révisions visibles sur la fig. 2 en découlent). En conséquence, la colonne calligraphiée est barrée verticalement et s'y substitue dans la colonne de gauche le texte récrit par Pietro.
- Lettres et manuscrits sont enfin complétés par un troisième support, des feuillets libres, qui transitent avec la correspondance ordinaire. C'est le cas lors de la révision des Meditazioni sulla economia politica, en novembre 1770. Sept jours après la réception du manuscrit relié, Alessandro renvoie ce dernier annoté, accompagné de dix-huit pages d'Osservazioni rédigées sur papier à lettres et conclues par un «Giudicio generale» comportant ce verdict : «à imprimer absolument 35 ». À la suite immédiate de ces

35. Voir Cart. IV, p. 74. Le texte des Osservazioni est édité dans Scritti di economia, finanza e amministrazione, t. II, éd. G. Bognetti et al., Edizione nazionale delle opere di Pietro Verri, II, Rome, Edizioni di Storia e Letteratura, 2007, p. 573-583. 


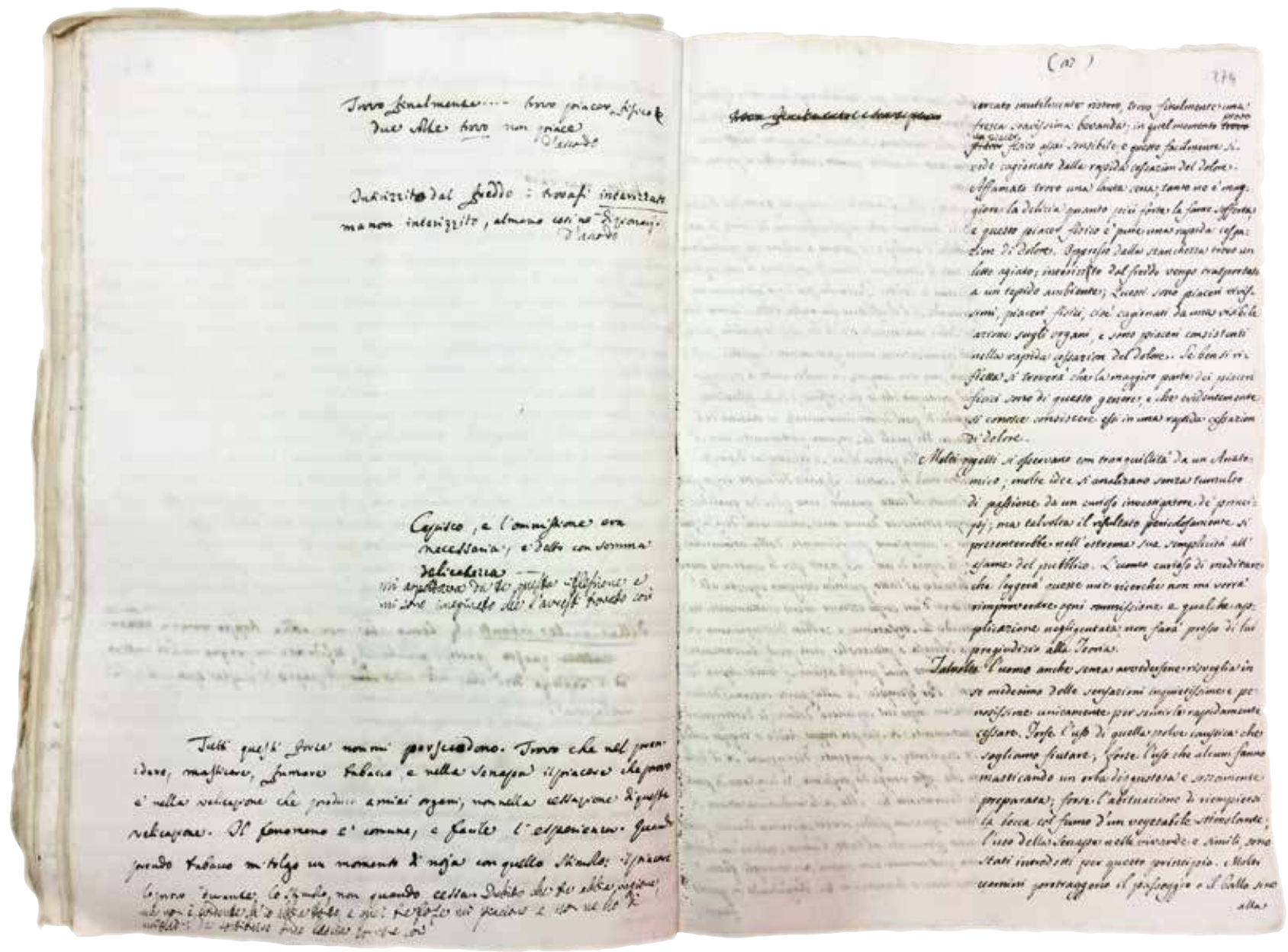

Fig. 3 : Archivio Verri, ibid., f. $273 \mathrm{v}^{\circ}-274 \mathrm{r}^{\circ}$

Exemples d'approbations apposées par Pietro, à l'encre légère, sous les observations et corrections d'Alessandro («D'accord»; «Je m'attendais à cette réflexion de ta part et je me suis imaginé que tu serais de cet avis»; «Je crains que tu aies raison, mais il n'est pas évident que j'aie tort»). Milan, Fondazione Raffaele Mattioli per la storia del pensiero economico.

(C) Photographie de P. Musitelli, avec l'aimable autorisation de la Fondazione Raffaele Mattioli per la storia del pensiero economico

observations, après un saut de ligne, commence la lettre datée du 21 novembre 1770 (fig. 4). Pour indiquer à quels éléments du texte il se réfère, Alessandro recopie sur ces pages le texte de Pietro (phrases ou mots soulignés), en indiquant la page et la ligne du manuscrit, puis la modification souhaitée, d'ordre conceptuel, linguistique ou syntaxique. Lorsque ces Osservazioni lui parviennent, Pietro en intègre le contenu jugé pertinent, parfois remanié, dans la colonne de gauche du manuscrit interfolié également annoté par son frère.

Le processus éditorial est ainsi englobé dans l'échange épistolaire. Une continuité s'instaure entre l'espace de la lettre et les autres supports de révision qui lui sont extérieurs à des degrés divers : témoin cette lettre inédite
d'Alessandro relative aux corrections de sa tragédie La Congiura di Milano classée par Pietro parmi le matériel préparatoire de la pièce qu'il révisa, lettre qui échappa aux éditeurs du tome $\mathrm{X}$ du Carteggio (Archivio Verri, dossier 494.2.1, lettre 1062 du 5 septembre 1778). Loin d'être une erreur d'archivage, c'est une preuve supplémentaire de la permutabilité des fonctions de ces différents supports manuscrits : le système dialogique de révision s'étend avec aisance tout à la fois sur les brouillons et sur les lettres, il se poursuit continûment de l'un à l'autre. Le dialogue épistolaire comme espace de genèse des textes, le manuscrit comme espace d'un dialogue épistolaire : les frontières sont perméables et la pensée circule. 


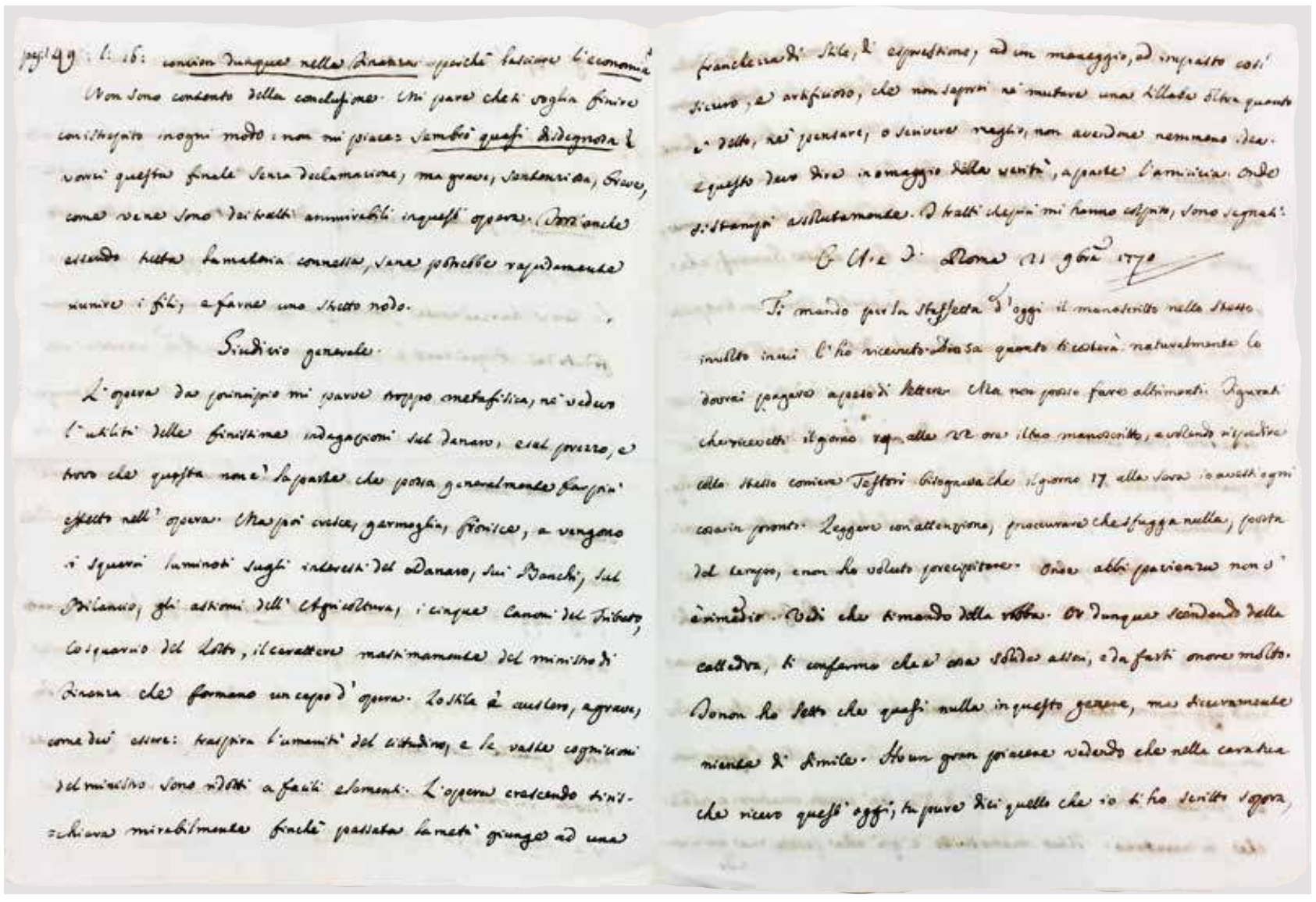

Fig. 4 : Archivio Verri, cartella 254, lettres autographes d'Alessandro Verri (1770), chemise 358 : «Lettera del 21 nov. 1770 colle "Osservazioni" », feuillet $\mathrm{V}$, double page centrale. Page de gauche : fin des Osservazioni et début du paragraphe «Giudicio generale». Page de droite : conclusion du précédent («si stampi assolutamente») et début de la lettre datée de Rome, 21 novembre 1770.

(c) Photographies de P. Musitelli, avec l'aimable autorisation de la Fondazione Raffaele Mattioli per la storia del pensiero economico

\section{Rêve d'homologie et singularisation des rôles}

La conviction qu'il existe une «parfaite analogie de sentiments 36 » entre les deux épistoliers constitue l'arrièreplan de ce processus. «Tu es un autre moi-même 37 », écrit Pietro à son frère en octobre 1771, faisant usage d'une figure répétée sept fois 38 entre 1766 à 1773 , et dont certaines occurrences se rattachent directement à la pratique de la révision. Chacun se berce de l'illusion d'avoir trouvé en son correspondant, frère de sang et de plume, l'autre «moitié de son âme» ( «Dimidium animae meae» écrit Alessandro ${ }^{39}$ ), selon l'allégorie classique de l'amitié, qui essaime d'Aristote à Cicéron et d'Érasme à Montaigne. Dans ce système gémellaire, la critique est d'autant mieux admise que for intérieur et for extérieur se confondent : le jugement de l'autre est un jugement qui vient de soi. Cela ne signifie pas pour autant que le processus de révision soit productif à la seule faveur d'une homologie de pensée. Au contraire, se fait jour une singularisation progressive de la voix et du rôle de chacun, dès le début des années 1770.

Admettant ne pas posséder l'expertise de son frère en matière d'économie et de finances publiques, Alessandro est enclin à formuler des observations d'ordre stylistique. Il s'en amuse à l'occasion de la deuxième série de révisions des Meditazioni sulla economia politica: «De ce sujet, je ne sais que ce que tu m'as appris. Et tu t'y consacres depuis des années. Pourtant tu n'as pas tort : Molière lisait ses comédies à sa servante 40 ». Pietro n'est pas moins prudent lorsqu'il avance avec circonspection ses remarques sur la versification et la distribution des

36. Cart. I/2, 9 juillet 1768, p. 348.

37. Cart. IV, 5 octobre 1771 , p. 250.

38. Cart. I/1, p. 87, 193 et $410 ;$ Cart. V, p. 116 ; Cart. VI, p. 68.

39. Cart. I/1, 29 mai 1767, p. 375.

40. Cart. IV, 1er décembre 1770, p. 83-84. 
actes de la Congiura di Milano: «Ne tiens pas compte de ce que je t'écris. Je me dédirai moi-même, sans doute; considère simplement mon âme qui se présente à toi sans voile ${ }^{41} »$. En réalité, les rôles respectifs de chacun se dessinent bientôt : le philosophe et le styliste. Lorsqu'il fait la «toilette 42 » des textes de son frère, Alessandro manifeste une exigence de correction formelle sans cesse plus marquée. En mars 1778, en relisant ce qui allait devenir le Discorso sulla felicità, il écrit :

Ce que l'on nomme «limer» une œuvre exige tant d'attention au détail qu'on en laisse volontiers le soin à un ami. [...] Je serai ton persécuteur pédant. J'examinerai avec ma fidélité spartiate tes nouveaux écrits, et je te dirai sévèrement mon avis, principalement en ce qui concerne le mécanisme du style 43 .

Dès 1769 , il avait reproché à Pietro certaines «expressions animées »dans son essai Sulle leggi vincolanti, l'invitant à «plus de modération» :

Le «bon ton» n'ôte pas la force des expressions : il l'accroît. Et il ne convient pas qu'un homme supérieur soit soupçonné d'animosité ou d'agacements particuliers, ni qu'il se départe jamais dans une affaire publique injustement traitée par les mauvais citoyens de la placidité d'une bienveillante philosophie 44 .

«Je crois que la renommée des livres ne vient pas de ce qu'ils disent, mais de la façon dont ils le font», lancet-il en septembre 1778, estimant qu'il vaut mieux «bien écrire » que « raisonner avec exactitude 45 ». Chacun cultive une conception opposée de la finalité des lettres et de sa relation à l'opinion publique : l'un veut convaincre et éclairer; l'autre sait plaire en cultivant ou en devançant les goûts littéraires. En conséquence, chacun envisage différemment le processus d'écriture. Cet écart, qui nourrit le débat épistolaire, ne porte pas préjudice, jusqu'à la fin des années 1770, à la productivité de leurs échanges. Après tout, comme l'observent Nicolas Donin et Daniel Ferrer, « un duo implique avant tout une différenciation des compétences et

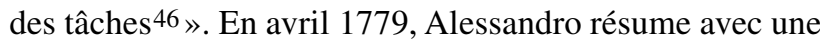
franchise désarmante :

Tu veux électriser, inspirer l'amour de la vérité, convertir les mauvais, réparer les têtes, et après t'être bien découragé, tu te rends compte que c'est du temps perdu. [...] Pour ma part, j'ai l'impression que la vérité est une bien belle chose, mais elle ne m'importe pas toujours 47 .
À l'orée de la décennie 1780, le clivage se creuse et finit par rendre inopérante la fonction génétique de la correspondance. Le dialogue fécond, qui reposait sur une affinité bâtie au cours des batailles éclairées de jeunesse, est mis à mal par l'apparition de divergences idéologiques et esthétiques. Comme l'a finement observé Bartolo Anglani, la fiction d'une «utopie "sentimentale" n'épuise pas toute la réalité du dialogue épistolaire » et retarde au contraire la prise de conscience, notamment chez Pietro, de «la réalité de deux existences guidées par une autonomie propre 48 ». Cette tension, ou «disanalogia», produit un déplacement de la gestation des textes hors de la correspondance.

\section{Les nouveaux horizons littéraires d'Alessandro Verri}

Il est frappant de constater qu'à partir du moment où Alessandro publie ses premiers textes romains, la correspondance devient un espace de cristallisation des oppositions. En 1778, après avoir achevé l'année précédente sa traduction d'Hamlet puis mené à bien celle d'Othello (Archivio Verri, dossier 491.3 et 4), il compose deux tragédies versifiées, publiées en 1779 à Livourne sous le titre Tentativi drammatici : Pantea, tragédie hellénisante inspirée de Xénophon, et la Congiura di Milano, drame politique moderne. Pietro est directement associé à la fortune éditoriale de ces textes, non seulement en qualité de relecteur et réviseur, mais pour avoir mis un copiste au service de son frère. Il fait exécuter en 1777 par le fils de son secrétaire Giorgio Ghelfi un manuscrit calligraphié d'Hamlet (Archivio Verri, 491.3.4). Il fait de même pour le manuscrit de la Congiura dont il reçoit de Rome une copie autographe à la fin de l'été 1778 . Il contribue également à la circulation de ces

41. Cart. IX, 20 juin 1778, p. 327.

42. Cart. II, p. 141 ; Cart. III, p. 46.

43. Cart. IX, 28 mars 1778 , p. 250.

44. Cart. II, 11 juillet 1769 , p. 363.

45. Cart. X, 9 septembre 1778, p. 71.

46. N. Donin et D. Ferrer, «Auteur(s) et acteurs de la genèse », Genesis, $\mathrm{n}^{\circ} 41,2015$, p. 12.

47. Cart. X, 7 avril 1779 , p. 236-237.

48. B. Anglani, «Il dissotto delle carte ». Sociabilità, sentimenti e politica tra $i$ Verri e Beccaria, Milan, Franco Angeli, 2004, p. 169. 
textes en Lombardie, en faisant notamment parvenir une nouvelle copie manuscrite des deux tragédies, calligraphiées «sur du beau papier de Hollande avec la belle écriture du jeune Ghelfi ${ }^{49}$ », à l'archiduchesse Marie Béatrice d'Este, protectrice des lettres.

Si la rhétorique de l'harmonie sentimentale dont chacun s'efforce d'entretenir la flamme survit encore dans les échanges consacrés aux révisions de ces textes, la connivence des deux frères se fragilise. D'une part, Pietro n'hésite pas à afficher sa préférence pour la Congiura di Milano au détriment du classicisme larmoyant de Pantea. Le drame historique, qui relate l'assassinat du duc Sforza de Milan en 1476, à l'instigation de Cola Montano, est inspiré des Histoires florentines de Machiavel et de L'Historia di Milano de Bernardino Corio. Saluant en ce texte «la

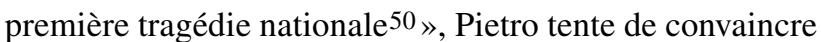
son frère de modifier la ligne politique et le sens de sa pièce, qui se clôt sur la condamnation des conjurés, promis à la torture, et celle du tyrannicide, prononcée par le conseiller du prince, Simonetti. «C'est ce que veut l'histoire», concède Pietro, qui ajoute : «Ta tragédie possède donc un vice dans son sujet ». Il aurait préféré qu'Alessandro s'éloigne de la chronique et suscite «hardiment» l'admiration du public pour l'action «absolument vertueuse » des jeunes conjurés. Aussi l'invite-t-il à renoncer au dernier acte et à clore sa tragédie sur le suicide du républicain Montano, en éliminant la mise en garde finale contre la tentation de la révolte : il fallait que l'avertissement valût tant pour les conjurés que pour les mauvais princes. Alessandro se raidit contre cette volonté de transformer son propos :

Je suis auteur, mais je suis aussi le sujet très fidèle de l'héritière puissante et bienheureuse de ce grand scélérat [Galeazzo Sforza], et le bien du principat, les devoirs modérés de la subordination qui lui est due exigent qu'on n'applaudisse jamais une conjuration, même contre Caligula ou Néron, car l'honnête homme doit alors se comporter comme Pomponius Atticus, c'est-à-dire aller vivre sous des cieux meilleurs, comme pouvaient le faire nos Brutus et Cassius lombards. Cette tirade de Simonetti, après les longues délibérations sur licet occidere tyrannum, sert de correctif, et il me semble que, tout bien considéré, l'auteur a insufflé plus de force dans les sentiments de liberté, que dans ceux de la tolérance ${ }^{51}$.

Pietro n'est déjà plus son unique interlocuteur. Alessandro a élargi le cercle de ses lecteurs milanais. Il dit avoir suivi pour réformer sa Congiura «le plan du comte Carli $52 »$. Ancien collaborateur des Pugni, et rival politique de Pietro, Gian Rinaldo Carli tend dans les années 1780-1790 à devenir le confident de ses expérimentations littéraires. Alessandro noue avec lui de forts liens d'estime et d'amitié. Tous deux partagent des convictions très conservatrices et critiques à l'égard notamment des Lumières françaises, dont Alessandro tarde à s'ouvrir avec franchise à son frère dans sa correspondance.

Par ailleurs, Alessandro s'est lancé dans la composition d'un roman archéologique néoclassique, Le Avventure di Saffo poetessa di Mitilene, dont la gestation rapide, entre juillet et novembre 1779, s'est faite à l'insu de son frère. D'où la surprise de ce dernier à la réception du manuscrit : «Tu es un ami singulier, cher Alessandro : tu me surprends avec des travaux toujours inattendus 53 ». Le seul manuscrit autographe conservé à Milan présente peu de ratures, quelques ajouts et corrections en marge ou à l'interligne, tous probablement autographes. Le manuscrit est disposé en pleine page, sans place en marge pour les ajouts, dans une écriture régulière sur des feuillets doubles reliés (fig. 5). Seuls trois feuillets (Archivio Verri, dossier 496.1, f. $89^{\circ} \mathrm{r}-91 \mathrm{r}^{\circ}$ ) présentent des révisions associées à des numéros de pages. Elles semblent également autographes, mais il pourrait s'agir d'une copie d'observations formulées par un relecteur autre que Pietro, car la correspondance n'en porte pas trace. On sait enfin grâce aux travaux d'Elisanna Pecoraro et d'Alfredo Cottignoli 54 qu'Alessandro mène a posteriori un inlassable travail de révision lexicale sur les premières éditions imprimées de son roman.

Parvenu à Milan en mai 1780, le manuscrit est fraîchement accueilli par Pietro, qui aurait préféré que son frère poursuive dans la voie de la tragédie historique. Il juge le texte «languido» et en interrompt les révisions. Alessandro s'en irrite à l'automne :

49. Cart. X, 17 octobre 1778, p. 106-107.

50. Cart. IX, 20 juin 1778, p. 325.

51. Cart. X, 30 janvier 1779, p. 184-185.

52. Lettre inédite du 5 septembre 1778, citée supra.

53. Cart. XI, 6 mai 1780, p. 66.

54. E. Pecoraro, «Le correzioni autografe del Verri alle Avventure di Saffo», Lettere italiane, 33/2, 1981, p. 222-234, et A. Verri, Le Avventure di Saffo, éd. A. Cottignoli, Rome, Salerno, 1991, «Nota al testo», p. $198-200$. 


\section{$\mathcal{S e p}_{2}$}

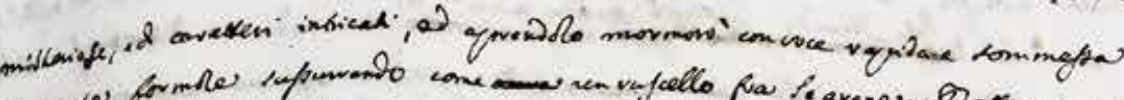

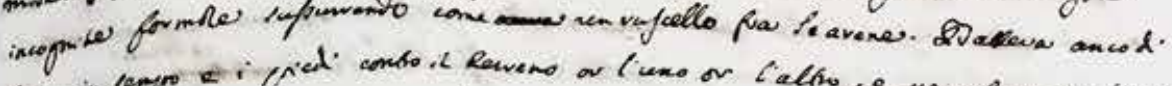

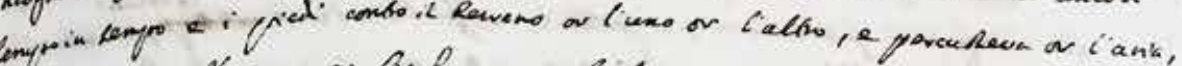

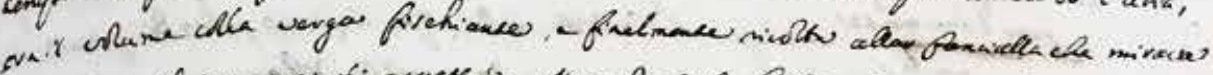

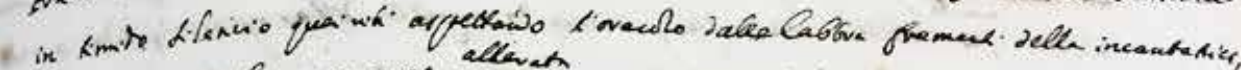

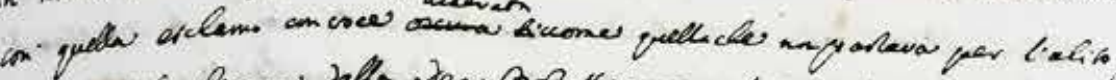

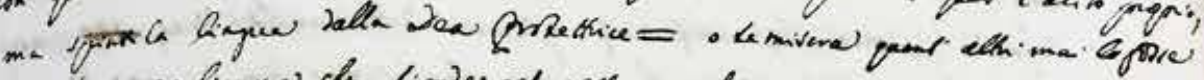

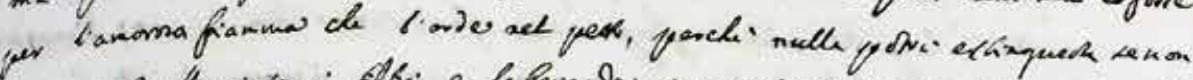

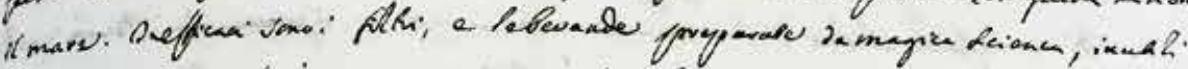

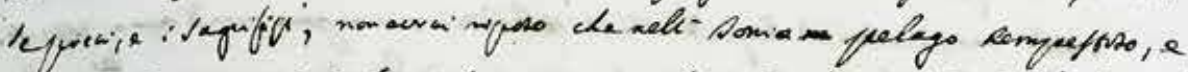

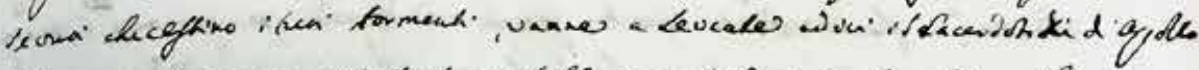

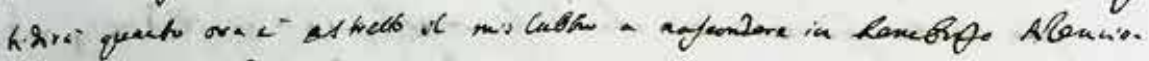

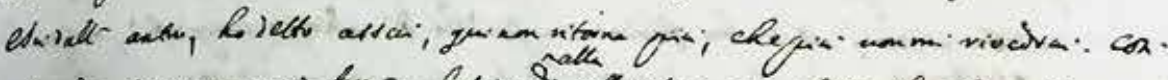

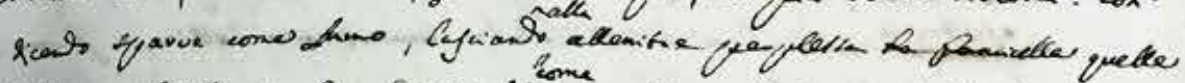

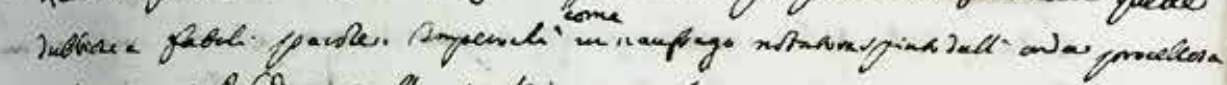

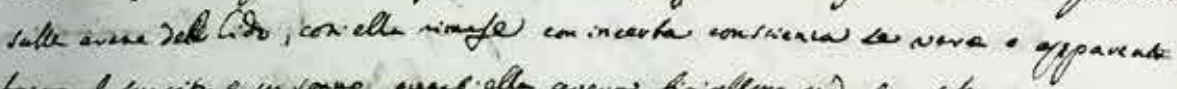

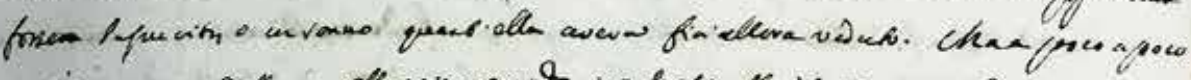

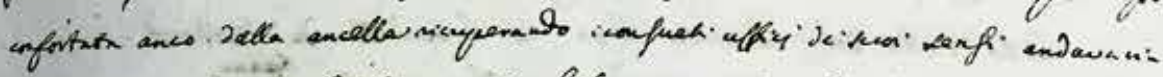

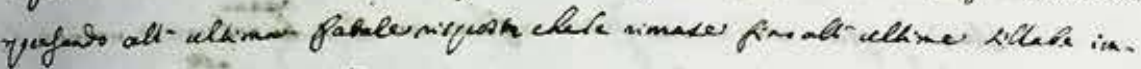

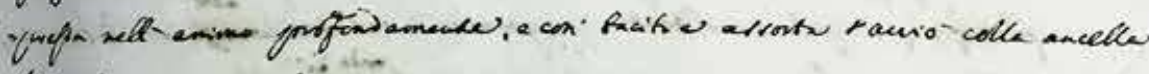

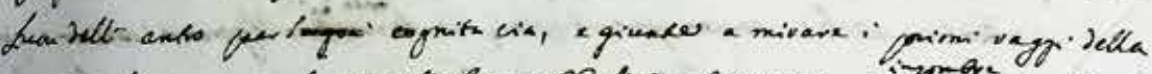

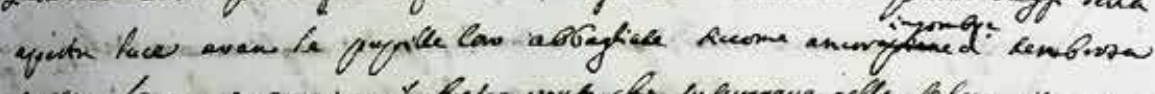

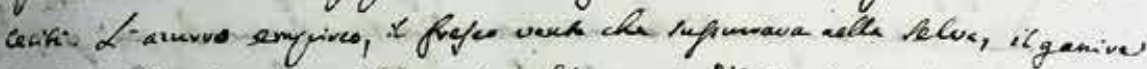

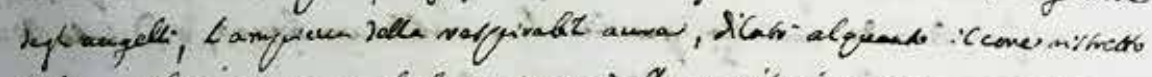

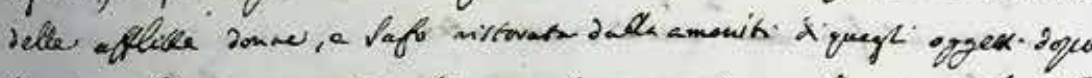

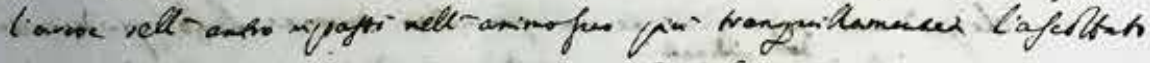

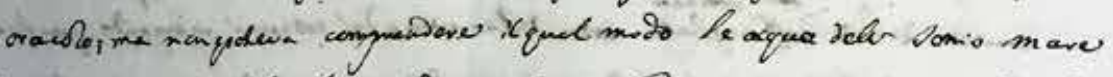

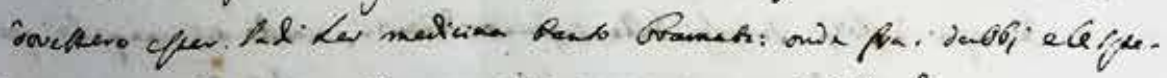

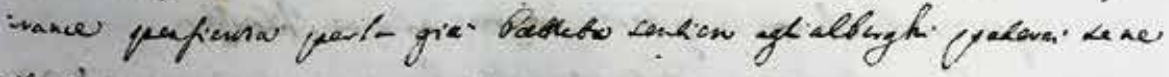
minine? okveramake

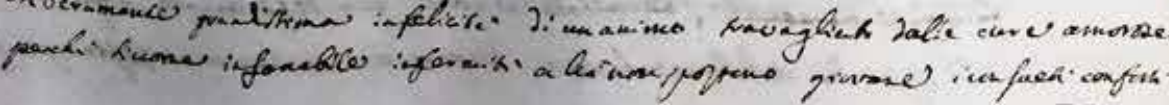

Fig. 5 : Milan, Archivio Verri, cartella 496, fasc. 1 : Le Avventure di Saffo, f. $39 \mathrm{r}^{\circ}$. De rares ajouts et corrections sont visibles à l'interligne. Manuscrit autographe d'Alessandro Verri, relié, composé entre le 20 juillet et le 20 novembre 1779 (indications en f. $1 \mathrm{r}^{\circ}$ et f. $67 \mathrm{v}^{\circ}$ ). La page ici reproduite contient la fin du chapitre «Le illusioni » (qui deviendra « Gli spettri » dans la rédaction finale) et l'incipit du chapitre «La fuga notturna».

(C) Photographie de P. Musitelli, avec l'aimable autorisation de la Fondazione Raffaele Mattioli per la storia del pensiero economico 
Tu ne me dis plus un mot des Avventure di Saffo [...]. Même s'il s'agit d'une œuvre médiocre, l'auteur n'accepte pas d'en être privé55.

Le mois suivant, il décide de faire rapatrier vers Milan tous les manuscrits de Pietro qui sont encore en sa possession, ainsi que ses lettres. C'est un geste important pour la constitution du vaste Archivio Verri, mais Pietro s'en attriste :

On dirait que tu éloignes de toi ce qui m'appartient. [...] Je vois que ton âme est blessée, et vivement blessée, car sa bonne foi aimante a été déçue $[\ldots]$; tu respires vengeance. Est-ce là le principe qui t'anime 56 ?

Le doute est instillé, la confiance est rompue.

En décembre 1780, Alessandro recouvre enfin son manuscrit, qui entre-temps a été lu avec enthousiasme par Carli et Beccaria, à qui Pietro l'a transmis, comme la Congiura di Milano en 1778. «Oh qu'elles sont belles les Aventures de Sapho!», s'exclame Carli le 8 juillet 1780, dans une lettre inédite présente dans le dossier du matériel préparatoire de Saffo (Archivio Verri, dossier 496.1. f. $93 \mathrm{v}^{\circ}$ ). Alessandro précisera à son frère, en janvier 1782 , qu'il avait donné le manuscrit à relire, à Rome, à «deux personnes » qui formulèrent une «sentence favorable 57 ». Pas un mot, en revanche, sur la suite du processus éditorial pourtant mouvementé : censure ecclésiastique, nécessité de retrancher des chapitres, édition sous fausse date de «Padoue, 1782», corrections apportées à l'édition originale, puis à la suivante de Vercelli en 1783, puis aux éditions de 1793 et de 1797, jusqu'à l'édition définitive de $1806 \ldots$ Alessandro mène désormais seul son activité d'écrivain. Pietro fait de même : l'un des derniers textes qu'il soumet au tribunal fraternel, la Storia di Milano, parvient sous la forme d'une copie soigneusement calligraphiée sur papier bleu de Hollande : «J'eus l'impression que l'auteur n'admettait pas beaucoup de ratures, aussi fis-je preuve de prudence 58 », confiait plus tard Alessandro qui, de relecteur, était devenu simple lecteur.

Si sa correspondance avec Alessandro est, tout au long des années 1770, un espace de mûrissement et de perfectionnement de la production éditoriale de Pietro, la réciproque est moins certaine. L'aîné suit certes avec intérêt l'évolution des goûts de son frère et son exploration des nouveaux territoires littéraires et esthétiques du Settecento finissant, d'Homère à Shakespeare, de Canova à Piranèse, de la tragédie au roman, mais force est de constater qu'Alessandro n'acquiert véritablement sa stature d'écrivain qu'au moment où la fonction génétique de leur correspondance s'enraye.

Entre 1784 et 1792 une violente querelle domestique et judiciaire déchire la fratrie autour de l'administration de l'héritage paternel. Les échanges s'interrompent presque totalement. Or, c'est précisément dans les années 1782-1790 qu'Alessandro mûrit, dans la foulée du succès de Saffo, son deuxième roman, Les Nuits romaines, promis à une immense fortune européenne. Il n'est pas exclu que l'affaiblissement de ses relations avec Pietro, qui exerçait un contrôle vigilant sur le contenu des textes de son frère, ait eu un effet libérateur. C'est en tout cas à Carli qu'Alessandro s'ouvrait de son projet, le 22 juin 1782, en des mots qu'il n'aurait sans doute pu écrire à son frère :

[...] j'hésite entre divers sentiers et l'abondance de la matière [1'histoire antique] fait qu'il m'est difficile de prévoir où me guidera la variété du sujet. En tout cas, je me propose de ne jamais compromettre ma tranquillité pour un livre, et surtout de ne jamais me fatiguer à le composer ${ }^{59}$.

Lorsque se renouent les relations avec Pietro, la correspondance des deux frères change de ton et d'objet. Elle devient le lieu où discuter des œuvres des autres - Alfieri, notamment - et des guerres révolutionnaires. Deux écritures, deux voix s'y répondent : celle du néoclassicisme conservateur et celle du réformisme républicain, qui s'y construisent l'une contre l'autre. Le temps et l'éloignement, conditions nécessaires à l'existence de la correspondance, ont fini par avoir raison de l'idée séduisante d'une infrangible union des cœurs, sous le charme de laquelle les deux frères ont vécu une quinzaine d'années. Le processus de création et de révision par voie épistolaire apparaît, rétrospectivement, comme l'ultime vestige d'une méthode de travail dont Pietro

55. Cart. XI, 14 octobre 1780, p. 172.

56. Cart. XI, 22 novembre 1780 , p. 190.

57. Cart. XII, 16 janvier 1782, p. 164.

58. Lettere di Alessandro Verri a Vincenza Melzi d'Eryl (1794-1816). Edizione e saggio di commento, éd. S. Rosini, Université de Pavie, 1998, lettre CLXXV du 8 novembre 1800.

59. Cité dans M. Udina, «A. Verri e G. R. Carli. Lettere inedite», Pagine istriane, VII, 1909, p. 14. 
Verri peine à faire le deuil, pris entre l'espoir de «rebâtir la Jérusalem» (l'Accademia de'Pugni) et le regret d'avoir vu se disperser l'ancienne société, «devenue comme le peuple israëlite $60 ! »$. Toute collaboration possède une dimension axiologique au sens où elle exige un ajustement réciproque des valeurs; la leur - malmenée par l'intensité avec laquelle chacun vivait et revendiquait la mission qu'il s'était assignée : le bien public, d'un côté, la résurgence du beau style italien de l'autre - ne fut plus en mesure de se poursuivre quand le compromis fit place à la confrontation.

60. Cart. I., 3 février 1767, p. 195-196; Cart. IV, 14 novembre 1770, p. 60.

PIERRE MUSITELLI est maître de conférences à l'École normale supérieure et membre de l'équipe «Écritures des Lumières » de l'ITEM (CNRS-ENS). Ses travaux portent sur la littérature et l'histoire du long XVIII ${ }^{\mathrm{e}}$ siècle italien, sur les Lumières lombardes, le néoclassicisme et la constitution du canon. Ouvrages récents : Le Flambeau et les ombres. Alessandro Verri, des Lumières à la Restauration (1741-1816), Rome, Collection de l'École française de Rome, 2016; Le bonheur du plus grand nombre. Beccaria et les Lumières, sous la direction de P. Audegean, C. Del Vento, P. Musitelli, X. Tabet, Lyon, ENS Éditions, 2017. 


\section{Genèses littéraires dans la correspondance de Pietro et Alessandro Verri}

La correspondance des frères Verri permet de suivre pendant trente ans (1766-1797) l'élaboration des principales œuvres économiques et philosophiques de Pietro et le mûrissement des goûts littéraires d'Alessandro au gré de multiples expérimentations de styles et de genres. Le premier vit à Milan, le second réside à Rome : tous deux développent, à travers leur correspondance hebdomadaire, une pratique collaborative de l'écriture. Brouillons et manuscrits transitent par voie postale pour être discutés et annotés, le texte prenant forme au fil de ces échanges. Mais l'écriture épistolaire des frères Verri n'a pas, en matière de genèse littéraire, qu'une fonction productrice : les jugements exprimés ne témoignent pas toujours d'une capacité de l'interlocuteur à saisir et déchiffrer l'œuvre et ses enjeux. Avec le temps, s'usent l'amitié fraternelle et ses promesses initiales (stimuler l'autre, être son censeur impartial), à mesure que deux styles s'affirment et que se creuse le clivage idéologique dans les années de la Révolution.

The correspondence of the Verri brothers makes it possible to follow the evolution, throughout thirty years (1766-1797), of the development of the most important economic and political works of Pietro, together with the development of Alessandro's literary taste through many experimentations with styles and genres. The first lived in Milan; the second in Rome: by their weekly correspondence, they developed a collaborative writing. Drafts and manuscripts are sent by post and are discussed and annotated, the text being shaped throughout these exchanges. But the correspondence of the Verri brothers does not only have, with regards to literary genetics, a productive function: the expressed opinions do not always reveal the counterpart's ability to understand the work and its issues. Over time, brotherly friendship vanishes and so does the initial commitment (to encourage the other, to be an unbiased censor), as two different styles are developed, and the ideological gap becomes wider in the years of Revolution.

Die Korrespondenz der Brüder Verri ermöglicht es, dreißig Jahre lang (1766-1797) die Ausarbeitung der wichtigsten ökonomischen und philosophischen Werke Pietros zu verfolgen, ebenso kann man die Reifung des literarischen Geschmacks von Alessandro, der mit verschiedenen Stilen und Genres experimentierte, beobachten. Der eine Bruder lebt in Mailand, der andere residiert in Rom: Durch ihre wöchentliche Korrespondenz entwickeln sie eine kollaborative Schreibpraxis. Entwürfe und Manuskripte werden zur Erörterung und Kommentierung per Post versandt, wobei der Text im Laufe dieses Austauschs Gestalt annimmt. Das Briefeschreiben der Brüder Verri hat jedoch nicht nur eine produktive Funktion mit Blick auf die literarischen Textgenese: Die getroffenen Urteile zeugen nicht immer von der Fähigkeit des Gesprächspartners, das Werk zu erfassen und seine Themen zu entschlüsseln. Mit der Zeit verbraucht sich die brüderliche Freundschaft mit ihren anfänglichen Versprechungen (den Anderen zu motivieren, ein unparteiischer Zensor zu sein), da sich zwei Stile entwickeln und sich die ideologische Spaltung in den Jahren der Revolution erweitert.
La correspondencia de los hermanos Verri permite seguir durante treinta años (1766-1797) la elaboración de las principales obras económicas y filosóficas de Pietro y la maduración de los gustos literarios de Alessandro a través de sus múltiples experimentaciones de estilos y géneros. El primero vive en Milán, el segundo reside en Roma; los dos desarrollan, mediante su correspondencia semanal, una práctica colaborativa de la escritura. Borradores y manuscritos transitan por vía postal para ser discutidos y anotados, y el texto toma forma a lo largo de estos intercambios. Sin embargo, la escritura epistolar de los hermanos Verri no cumple, en materia de génesis literaria, solo una función productora: los juicios expresados no siempre dan cuenta de la capacidad del interlocutor para comprender y descifrar la obra y sus desafíos. Con el tiempo, la amistad fraterna y sus promesas iniciales (estimular al otro, ser su censor imparcial) se deterioran a medida que los dos estilos se afirman y que se profundiza la brecha ideológica en los años de la Revolución.

A correspondência dos irmãos Verri nos permite acompanhar, durante 30 anos (1766-1797), a elaboração das principais obras econômicas e filosóficas de Pietro e o amadurecimento dos gostos literários de Alessandro a partir de diversas experimentações de estilos e de gêneros. O Pietro vive em Milão, Alessandro, em Roma : ambos desenvolvem através das suas correspondências semanais, uma prática de escrita colaborativa. Rascunhos e manuscritos transitam por via postal para serem discutidos e anotados, enquanto o texto vai ganhando forma ao longo dessas trocas. Mas a escrita epistolar dos irmãos Verri não tem, em matéria de gênese literária, apenas uma função produtora : os julgamentos expressos nem sempre testemunham, da parte do interlocutor, uma capacidade de compreender e de decifrar a obra e seus desafios. Com o tempo, a amizade fraterna se deteriora e suas promessas iniciais (estimular o outro, ser o seu censor imparcial) se perdem, na medida em que dois estilos distintos se afirmam e que se aprofunda a clivagem ideológica nos anos da Revolução.

La corrispondenza tra i fratelli Verri permette di seguire, nell' arco di trent'anni (1766-1797), l'elaborazione delle principali opere economiche e filosofiche di Pietro e la maturazione dei gusti letterari di Alessandro attraverso varie sperimentazioni di stili e di generi. Il primo vive a Milano, il secondo a Roma: entrambi sviluppano, tramite la corrispondenza settimanale, una pratica collaborativa della scrittura. Abbozzi e manoscritti circolano a mezzo posta per essere discussi e annotati, e il testo prende progressivamente forma attraverso questi scambi. Ma, dal punto di vista della genesi letteraria, la scrittura epistolare dei fratelli Verri non ha soltanto una funzione produttiva: i giudizi espressi non sempre testimoniano della capacità, da parte dell'interlocutore, di comprendere l'opera e di coglierne la posta in gioco. Con il passare del tempo l'amicizia fraterna si affievolisce e le sue iniziali promesse (stimolare l'altro, essere il suo censore imparziale) vengono meno, via via che due stili distinti si affermano e che si approfondisce il divario ideologico negli anni della Rivoluzione. 